\title{
Inhibition of JAK3/STAT3 Activation in Rats Uterine Leiomyoma by formula of Taohong Siwu Decoction: A Randomized Controlled Trial
}

\section{Li Luo}

Chengdu University of Traditional Chinese Medicine Wenjiang Campus: Chengdu University of Traditional Chinese Medicine

\section{Shasha Shi}

Chengdu University of Traditional Chinese Medicine Wenjiang Campus: Chengdu University of

Traditional Chinese Medicine

\section{Li Zhang}

Chengdu University of Traditional Chinese Medicine Wenjiang Campus: Chengdu University of

Traditional Chinese Medicine

\section{Xun Xu}

Chengdu University of Traditional Chinese Medicine Wenjiang Campus: Chengdu University of

Traditional Chinese Medicine

\section{Fu Peng}

Sichuan University

Chenghao Yu ( $\nabla$ yuholy@126.com )

Chengdu University of Traditional Chinese Medicine https://orcid.org/0000-0002-5793-1864

Liangwwen Li

Chengdu University of Traditional Chinese Medicine Wenjiang Campus: Chengdu University of

Traditional Chinese Medicine

Tie Zheng

Tsinghua University

\section{Research}

Keywords: Uterine leiomyoma, Taohong Siwu Decoction, JAK3, STAT3, Immune checkpoi

Posted Date: August 10th, 2021

DOI: https://doi.org/10.21203/rs.3.rs-786708/v1

License: (c) (i) This work is licensed under a Creative Commons Attribution 4.0 International License.

Read Full License 



\title{
1 Inhibition of JAK3/STAT3 activation in rats Uterine
}

\section{Leiomyoma by formula of Taohong Siwu Decoction: A}

3 randomized controlled trial

4 Li Luo ${ }^{1}$, ShaSha Shi ${ }^{1}$, Li Zhang ${ }^{1}$, Xun $\mathrm{Xu}^{2}$, Fu Peng ${ }^{3 *}$, ChengHao Yu${ }^{1,4^{*}}$, LiangWen Li ${ }^{1}$, Tie

5 Zheng ${ }^{5}$

$6{ }^{1}$ Basic Medicine College, Chengdu University of Traditional Chinese Medicine, Chengdu, China

$7 \quad{ }^{2}$ Basic Medicine College, Yunnan University of Traditional Chinese Medicine, Yunnan, China

$8 \quad{ }^{3}$ West China School of Pharmacy, Sichuan University, Sichuan, China

$9 \quad{ }^{4}$ State Key Laboratory of Southwestern Chinese Medicine Resources, Sichuan, China

${ }^{5}$ School of Environment, State Key Joint Laboratory of Environment Simulation and Pollution Control,

11 Tsinghua University, Beijing, China

* Correspondence:

Fu Peng

fujing126@yeah.net.

Chenghao $\mathrm{Yu}$

yuholy@126.com.

\begin{abstract}
Background: Traditional Chinese medicine (TCM) formula of Taohong Siwu decoction (THSW) is often used in traditional Chinese medicine for the treatment of uterine leiomyoma (UL). The effectiveness of THSW for the treatment of UL has been confirmed in previous studies. At present, there are few relevant mechanism studies. The purpose of this study is to explore the efficacy and mechanism of THSW in the treatment of uterine leiomyoma.

Methods: A Sprague Dawley (SD) rat model of UL was established via estrogen and progesterone load combined with external stimulation for 5 weeks. We randomly assigned adult female non-pregnant rats into six groups: a control group, a UL model group, and a positive drug group,THSW(18g/ml) group, THSW $(9 \mathrm{~g} / \mathrm{ml})$ group, THSW $(4.5 \mathrm{~g} / \mathrm{ml})$ group. THSW group and positive drug group were treated with THSW medicinal solutions and gongliuxiao capsule medicinal solutions respectively after daily modeling for 3 weeks. Histological analyses, Polymerase Chain Reaction and western blotting were performed to evaluate the effect of THSW on UL and elucidate its mechanism of action.

Results: The uterus morphology of the model group showed significantly more swelling than that of the control group, and pathological of changes of rats were obvious in the model group. Compared with
\end{abstract}


model group, the pathological changes of UL were relieved in the THSW group. Our data showed that the treatment of rats with THSW significantly reduced the expression of cytotoxic T lymphocyte associated antign 4 (CTLA4) and indoleamine 2, 3-dioxygenase 1 (INDO). The phosphorylation of JAK3 and STAT3 which are the main signal transduction molecule of cytokines and growth factorswere were also inhibited.

Conclusions: Our results suggest that THSW is effective in the prevention and treatment of UL in rats, and THSW may exert its functions by inhibiting the activation of Tumor-related signal transduction pathway (JAK3/STAT3), immune escape, proliferation of tumor cell and improving apoptosis.

\section{Keywords: Uterine leiomyoma, Taohong Siwu Decoction, JAK3, STAT3, Immune checkpoint}

\section{INTRODUCTION}

Uterine leiomyoma, also known as uterine fibroma, is the most common benign tumors in women of child-bearing age, mainly caused by the proliferation of smooth muscle cells [1]. The incidence of the UL is on the rise, and the population of the UL is gradually younger [2]. The incidence rate of women was $20.0 \%-25.0 \%$ and $25.0 \% \sim 50.0 \%$ of patients showed clinical symptoms [3], such as, abnormal uterine bleeding, lower abdominal pain, and even infertility or abortion. A very small number of UL malignant could become sarcoma, accounting for about $0.5 \%$ of uterine fibroids [4].Studies have shown that women with UL are usually more likely than normal women to feel depressed and restless, and have a higher risk of depression [5], which seriously affects women's physical and mental health. The global economic expenditure caused by UL is about \$5.9-34.4 billion annually [6]. UL have become a problem related to health and socio-economic all over the world. Due to the lack of understanding of the underlying pathobiology, there is currently no fine medical treatment [7-9].The current medical treatment of UL is mainly divided into surgical resection and drug treatment. Currently, the mainstream theory believes that UL is a hormone-dependent tumor. Therefore, the medical treatment of UL mainly is inhibiting steroid hormones at present, which has certain effect, but once the use is stopped, there is a high tendency of recurrence. About three quarters of UL are treated with hysterectomy [10]. Studies have shown [11] that there is $\sim 28 \%$ risk of surgical complications. In some studies [12], the recurrence rate of surgery was more than $62 \%$.In the United States, the lifetime risk of myomectomy is $45 \%$ higher [13]. Therefore, the conventional Western medicine has the characteristics of high recurrence rate, a lot of side effects and many complications at present. In fact, only $10 \%$ to $20 \%$ of UL patients need surgery. Therefore, it is important to find an effective and safe treatment for UL. Replacement therapy such as TCM may eliminate the need of surgery treatment [14]. In this study, we used a rat model of qi stagnation and blood stasis UL, in which UL was induced via estrogen and progesterone load combined with external stimulation [15]. This model is more consistent with the pathological of human uterine leiomyoma.

Signal transduction, apoptosis and proliferation are one of the focuses of modern oncology. In addition, the mechanism of excessive proliferation in uterine smooth muscle cells and deposition of extracellular matrix is tightly regulated by a complex network, including cytokines, growth factors, MMP and TGF- $\beta$ etc.. Current studies have shown that JAK3 belongs to a member of non-receptor tyrosine protein kinase family. and is mediated by cytokine and growth factor-initiated signals via the JAK/STAT pathway. JAK3 is mainly expressed in hematopoietic cells and immune cells, as well as in vascular cells and cancer cells, and is involved in cell proliferation, apoptosis and immune regulation 
[16] It is a key mediator in regulating cells of metabolism and development and immune response $[17,18]$. In addition, JAK3 can affect the occurrence and development of diseases through various ways. The overexpression of JAK3 can induce vascular smooth muscle proliferation [19]. Sustained activation of JAK/STAT signal leads to increased expression of MMP [20]. These pathways are closely related to the formation of UL.

STAT3 is an important part of signal transduction and transcriptional activation, which plays an important role in the process of apoptosis and angiogenesis. It can mediate the interactive dialogue between tumor cells and immune cells, and is related to tumor invasion, metastasis, and immune escape. STAT3 plays a key role in tumor, including inhibition of apoptosis, alteration of cell cycle, and mediating angiogenesis and immunosuppression. Studies have shown that STAT3 can inhibit anti-tumor immunity by up-regulating apoptosis suppressor genes. STAT3 can also inhibit the pro-inflammatory cytokines produced by tumor cells, contribute to immune escape and promote the occurrence of tumor [21]. Continuous activation of STAT3 can promote tumor neovascularization $[4,22]$ In addition, JAK / STAT pathway can regulate growth factors such as VEGF, TGF, IGF and matrix metalloproteinases to promote cell proliferation and survival and inhibit apoptosis, which is closely related to the occurrence and development of UL [23]. There is now increasing evidence to support the important role of the STAT in tumors [24-26]. Therefore, there is a 3theoretical basis for exploring the signal transduction and transcriptional activation of JAK/STAT. However, there are few studies on the expression of JAK/STAT in UL at home and abroad. Because excessive proliferation of smooth muscle cells leads to the development of UL, we hypothesized that UL can be inhibited by inhibiting JAK3 / STAT3 activation.This study preliminarily explored the expression of JAK/STAT in UL, providing evidence for further study on the pathogenesis of UL. The results of this study will provide experimental basis for the treatment of UL with TCM.

Uterine fibroids in TCM theory is considered to be caused by stagnation of Qi and blood. Promoting blood circulation and metabolism is the fundamental method of treating UL in TCM [22,27]. Studies have shown that peach kernel, red peony root, poria cocos, zedoary turmeric, peony bark, sparganium, angelica, cyperus, cinnamon twig and oyster are the most frequently used for the treatment of UL, most of which are used for promoting blood circulation and removing blood stasis. Modern pharmacological studies have confirmed that the herbs which can promote blood circulation can improve hemorheological properties [28], thus reducing the size of UL. THSW is composed of peach kernels, safflower, peony, chuanxiong, dried ground and angelica. It has the effect of promoting blood circulation and tonifying qi and blood. It has a history of thousands of years and has good clinical efficacy in the treatment of gynecological diseases such as uterine fibroids and endometriosis. Modern pharmacological studies have confirmed that THSW has effects such as improving blood status, regulating immune function, regulating uterine smooth muscle, anti-oxidation, anti-inflammation, anti-cancer, anti-fibrosis and so on [29]. Shaoyun Li [30] found that THSW in the treatment of UL can significantly improve the therapeutic effects and reduce the size of fibroids. In addition, THSW can effectively improve the symptoms related to UL and improve the quality of life of patients with almost no side effects. Although THSW show a good effect in the treatment of UL in clinical, its mechanism of action is still unclear due to the characteristics of multi-channel and multi-target of TCM. Therefore, it is of great significance to understand the above signal pathway and related protein expression as one of the pathways of THSW in the treatment of UL. 


\section{MATERIALS AND METHODS}

\section{Preparation of Herbal Decoction}

119 The medicinal materials (peach kernel, safflower, chuanxiong, red peony root, cooked ground, and angelica $=1: 1: 1: 1: 1)$ (Kangmei Pharmaceutical Co., Ltd, 200101811) were crushed, soaked in water for $2 \mathrm{~h}$, and the residue was decocted twice: $1 \mathrm{~h}$ for the first extraction, $0.5 \mathrm{~h}$ for the second extraction. After the mixture was mixed and filtered, the solution was concentrated to $18 \mathrm{~g} / \mathrm{ml}, 9 \mathrm{~g} / \mathrm{ml}, 4.5 \mathrm{~g} / \mathrm{ml}$ separately and stored at $4^{\circ} \mathrm{C}$.The medicine in gongliuxiao capsule (Shandong step size Shenzhou Pharmaceutical Co., Ltd, z20055635) was poured into the mortar, ground thoroughly, dissolved with appropriate amount of ultrapure water, and mixed evenly to obtain $0.09 \mathrm{~g} / \mathrm{ml}$ medicine liquid, which was put in the refrigerator at $4{ }^{\circ} \mathrm{C}$ for standby.

\section{Rats}

Rats were cared for and tested in accordance with the plan approved by the Animal Ethics Committee of Chengdu University of Traditional Chinese Medicine. Female SD rats without specific pathogen [animal license number SYXK (Chuan) 2019-049] were purchased from Animal Experimental Center of Chengdu University of Traditional Chinese Medicine (China). All experiments were approved by the Animal Health and Use Committee of Chengdu University of Traditional Chinese Medicine. All animals were anesthetized with $2 \%$ sodium pentobarbital $(0.25 \mathrm{~mL} / 100 \mathrm{~g})$ and killed through cervical dislocation after 5 weeks. We monitored the diet, weight, and general behavior of the rats weekly.

\section{Study Design}

138 Forty-two SD rats(220-250g) were randomly divided into 6 groups: control group(n=7), model $\operatorname{group}(\mathrm{n}=7)$, positive drug $\operatorname{group}(\mathrm{n}=7), \operatorname{THSW}(18 \mathrm{~g} / \mathrm{ml}) \operatorname{group}(\mathrm{n}=7), \operatorname{THSW}(9 \mathrm{~g} / \mathrm{ml}) \operatorname{group}(\mathrm{n}=7)$, THSW $(4.5 \mathrm{~g} / \mathrm{ml})$ group $(\mathrm{n}=7)$. Rats were kept in the Center of Laboratory Animals at Chengdu University of TCM. Gongliuxiao capsule was used as the positive drug. The UL model was established on the basis of adaptive feeding. The UL model was established using methods previously validated(11). Rats in the control group received no treatment. The other 5 groups were given intramuscular injection of estradiol benzoate (Ningbo No.2 hormone factory, 2003251) with $0.5 \mathrm{mg} / \mathrm{kg}$ daily and intramuscular injection of progesterone (Ningbo No.2 hormone factory, 2003242) with $1 \mathrm{mg} / \mathrm{kg}$ every two days for 5 weeks. From the fourth week, the rats in the five experimental groups were subcutaneously injected with adrenaline hydrochloride (Ningbo No.2 hormone factory, 200311) with $0.5 \mathrm{mg} / \mathrm{kg} / \mathrm{d}$, and $4 \mathrm{~h}$ after injection, external stimulation was applied once: (1) day and night reversal, (2) continuous stimulation with $60 \mathrm{~dB}$ noise for $3 \mathrm{~h}$, (3) continuous upside down for 10 minutes, and (4) water bath at $5-10^{\circ} \mathrm{C}$ for 4 minutes. This went on for two weeks; Each stimulus was applied $\geq 2$ times over 2 weeks. At the beginning of modeling, the drugs $(10 \mathrm{ml} / \mathrm{kg})$ were administered simultaneously to the intervention group by gavage and to the appropriate group separately. The control group was given distilled water (Sichuan Kelun Pharmaceutical Co., Ltd, L218070808)2ml daily. The rats in the model group were not given drug intervention. After 5 weeks, the rats were anesthetized and the uterus was removed.(Figure1)

\section{Specimen Harvest}


At the end of the experiment, the rats were sacrificed after an overnight fast, the rats were anesthetized by $2 \%$ sodium pentobarbital $(0.25 \mathrm{~mL} / 100 \mathrm{~g})$. We stripped the uterus, removed the surface adipose tissue, removed the blood with normal saline, and then measured. We measure the transverse diameter and diameter with vernier calipers. After measurement, we divided the uterus into three parts and put them into the corresponding fixative.

\section{Histological Analyses}

It was embedded in paraffin, sectioned, blocked with a blocking buffe and stained. Then it was stained with hematoxylin (Wuhan Google Biotechnology Co., Ltd, G1005) for 3-7 minutes, and then stained with eosin (Wuhan Google Biotechnology Co., Ltd, G1005) for 4 minutes. The uterus was fixed overnight in $4 \%$ paraformaldehyde. The sections images were analyzed with a digital photographic microscope (BA400Digital; Mike Audi Industry Group Co., Ltd.).

\section{Western Blotting}

The uterine tissue was ground into homogenate and centrifuged. The supernatant was taken for testing, and the protein was detected using the BCA protein quantitative kit (Hangzhou Lianke Biotechnology Co. Ltd, 70-PQ0012). The PVDF membrane (0.45um)(millipore, IPVH00010) was used to transfer the membrane. After incubation by 1:3000 primary antibody of CTLA4 (anti-rabbit, bioss, bs-10006R), INDO (ati-rabbit, Affinity, DF6502) for 4h, the protein was incubated again by 1:3000 secendary antibody (Hangzhou Lianke Biotechnology Co., Ltd, 70-GAR007) labeled with HRP for 30min. Finally, chemiscope analysis was used for gray analysis. $\beta$-actin was selected as the loading control for protein expression analyses.

\section{Polymerase Chain Reaction}

Total RNA in the uterine tissue was extracted via TRLzol (invitrogen, 15596018). Reverse-transcription of mRNA was performed using the PrimeScript RT Reagent Kit with gDNA Eraser. Real-time PCR experiments were performed using the SYBR Green master mix (ABM, 019484830001). GAPDH was selected as the loading control for mRNA expression analyses. The corresponding primers for detecting JAK3, STAT3 and GAPDH are listed in Table S1.

\section{Statistical Analysis}

All data were statistically analyzed by SPSS 23.0. We used one-way analysis of variance (ANOVA) if the variances are homogeneous. Otherwise, nonparametric validation is used. The results are expressed as mean \pm standard deviation. We considered $p<0.05$ as statistically significant.

\section{RESULTS}

\section{Establishment of the Rat Model}

In control group, all rats were in good physical and mental health. The rats in the model group showed large area of hair loss and weight loss, reduced food intake, or hyperactivity. The transverse and longitudinal diameters of the uterus in the model group increased, and the size of UL also increased significantly(Figure2 and Figure3). Pathological examination showed that compared with the control group, part of the endometrium epithelial cells in the model group were necrotic and abscisic, with 
local smooth muscle cells hyperplasia and disordered arrangement, accompanied by a small amount of neutrophil infiltration. The uterus of rats in the model group (Figure 4B) was dull and transparent, with asymmetry and uneven thickness on both sides of the uterus. Also the uterus of rats in the model group was serious distortion and deformation, and obvious edema and nodules. Compared with the model group, the pathological state described above in the intervention group was improved to varying degrees.

\section{Effects of THSW on the Appearance of the Uterus in Rats}

Compared with the model group (Figure 4B), the uterine edema of rats in intervention group (Figure 4C-4E) was alleviated. Most of uterus were symmetrical and uniform thickness. Compared with the model group, the texture became soft and the surface of uterus was more smooth, and the volume of uterus became smaller, except for the THSW $(4.5 \mathrm{~g} / \mathrm{ml}$ ) group (Figure $4 \mathrm{~F}$ ). It may be that low-dose drugs have less therapeutic effect.

\section{Effects of THSW on Histological Changes of the Uterus}

In the model group (Figure 5B) the endometrial epithelial cells were more necrotic and exfoliated. Local uterine smooth muscle cells were hyperplastic and disordered with a small amount of neutrophil infiltration. In the positive drug group (Figure 5C), a few endometrial epithelial cells were necrotic and neutrophils in lamina propria were increased. The smooth muscle cells of the myometrium was regularly arranged. Increased neutrophils were seen in the lamina propria in THSW $(18 \mathrm{~g} / \mathrm{ml})$ group (Figure 5D). The smooth muscle cells of the myometrium were arranged slightly irregularly with only a small amount of neutrophils infiltration in the lamina propria. A large number of neutrophils were seen in the lamina propria in THSW $(9 \mathrm{~g} / \mathrm{ml})$ group (Figure 5E). Local smooth muscle cells were hyperplastic and regularly arranged. Increased neutrophils were seen in the endometrium lamina propria in THSW $(4.5 \mathrm{~g} / \mathrm{ml})$ group (Figure $5 \mathrm{~F})$. Local smooth muscle cells were hyperplastic and disordered. These results suggest that THSW $(18 \mathrm{~g} / \mathrm{ml})$ and THSW $(9 \mathrm{~g} / \mathrm{ml})$ can significantly improve the histological status of UL.

\section{Effect of THSW on activation of JAK3 and STAT3 of the Uterus}

Fluorescence quantitative PCR analysis showed that the expression of JAK3 in model group was significantly increased compared with control group $(P<0.01)$. Compared with the model group, the expression of JAK3 was also decreased in $\operatorname{THSW}(4.5 \mathrm{~g} / \mathrm{ml})$ group, but the difference was not statistically significant $(P>0.05)$. The effect of THSW on the expression of JAK3 was dose-dependent, so the THSW $(4.5 \mathrm{~g} / \mathrm{ml})$ had a weak inhibitory effect on the overactivation of JAK3. Compared with the model group, the expression of JAK3 was significantly decreased after treatment with THSW $(18 \mathrm{~g} / \mathrm{ml})$ and THSW $(9 \mathrm{~g} / \mathrm{ml})(P<0.001)$. The positive control group had similar inhibitory effect on JAK3 overactivation $(P<0.01)$. (Figure 6$)$

Compared with the control group, the expression of STAT3 in the model group $(P<0.001)$ was significantly increased, and the difference was statistically significant. Compared with the model group, the expression of STAT3 in the positive drug group $(P<0.05)$ and THSW $(9 \mathrm{~g} / \mathrm{ml})(P<0.05)$ was significantly decreased, and the difference was statistically significant. The expression of STAT3 in THSW $(18 \mathrm{~g} / \mathrm{ml})$ group $(P>0.05)$ and THSW $(4.5 \mathrm{~g} / \mathrm{ml})$ group $(P>0.05)$ had a decreasing trend, but the 
difference was not statistically significant (Figure 7).

\section{Effect of THSW on the Expression of INDO/CTLA4 in the Uterus}

245 Western blot analysis showed that compared with model group, the expression of INDO was significantly decreased after treatment of THSW $(P<0.05)$. The expression of INDO in model group was significantly higher than that in control group $(P<0.01)$. In the positive group, the expression of INDO was similar to that in the drug intervention groups $(P<0.01)$ (Figure 8B). Western blot analysis showed that compared with the model group, the expression of CTLA4 in uterine of the drug intervention group and the positive drug group was significantly decreased, but the difference was not statistically significant $(P>0.05)$. The effect of CTLA4 in the positive group and the drug intervention group was similar. Compared with the control group, CTLA4 was significantly increased in the model group, however, the difference was not statistically significant $(P>0.05)$. It may be because the drug intervention time is not enough. (Figure $8 \mathrm{C}$ )

\section{DISCUSSION}

At present, researches on the etiology and pathogenesis of uterine fibroids at home and abroad mainly include: sex hormones and receptors, cell proliferation and apoptosis, abnormal expression of proto oncogenes, signal transduction, molecular genetics and related gene expression, MMP etc. [31]. At present, the mainstream theory believes that UL are estrogesterone-dependent tumors. Studies have shown that the role of hormones in cell proliferation and differentiation is regulated by a variety of growth factors. Due to the involvement of JAK / STAT in the signal transduction pathways of a variety of growth factors and hormones, the abnormal activation of this pathway is closely related to the occurrence, development and prognosis of a variety of diseases. However, as far as we know, no report has evaluated the effect of THSW on the expression of JAK3 and STAT3 in UL. Therefore, in this study, we aimed to address this issue using a well-established rat model of UL. Morphological and pathological data showed that THSW treatment significantly improved the symptoms of UL. Therefore, we further investigated whether THSW can improve UL by inhibiting the activation of JAK3 and STAT3. Activation of JAK3 leads to the occurrence and development of many tumors. Studies have shown that [32] inhibiting the phosphorylation of JAK3 can reduce the activity of tumor cells. Wang Yiqi and Liu Minghua et al. $[33,34]$ found in their studies that TCM can reduce the proliferation and migration of tumors by inhibiting the JAK/STAT3 pathway, promote cell apoptosis, and inhibit the migration and invasion of tumor cells. Shushan et al. [35] also found that the addition of a tyrosine kinase inhibitor to uterine fibroid cells in vitro culture significantly inhibited the activation of STAT3, which in turn inhibited the proliferation of uterine fibroid cells, suggesting that STAT3 is a potential biomarker of UL. In addition, some studies have found that [36] activated STAT3 can improve the transcriptional activity of estrogen receptor and progesterone receptor, which may be involved in the pathological process of uterine fibroids. In Li Cairong's study [37], STAT3 was found to be involved in the regulation of proliferation and differentiation of uterine fibroids, tumor angiogenesis and cell cycle etc.. In fact, the results of real-time PCR showed that in each group of THSW, the expression of JAK3 and STAT3 in utero was significantly reduced, indicating that JAK3 and STAT3 could be inhibited by THSW, which inhibited tumor proliferation and promoted apoptosis. 
escape are a class of inhibitory receptors located on the surface of immune cells. Their function is to prevent autoimmunity caused by excessive activation of immune function, which is utilized by tumor cells, and tumor cells produce corresponding ligands to bind to it [38]. Subsequently, the activity of immune cells can be reduced. Studies have found that overexpression of JAK / STAT pathway can promote the expression of immune checkpoint. CTLA4 and INDO are immune checkpoints that have been studied deeply in recent years. INDO can directly inhibit the immune activity of $\mathrm{T}$ cells, or indirectly inhibit the activity of T cells by accelerating tryptophan depletion [39]. CTLA4 can block the pathway of $\mathrm{T}$ cell activation and has a negative regulatory effect on $\mathrm{T}$ cell activation [40]. Immune checkpoints are closely related to the occurrence and development of tumors. In the experiment of Sho et al. [41], it was demonstrated that CTLA4 plays an important role in inducing immune tolerance. Rigas et al. [19] injected complete anti-human CTLA4 MABCP-675, 206 intravenously into tumor patients and observed that the anti-tumor immune effect in patients was increased and the disease was controlled. Some studies have also found [32] that TCM can treat uterine fibroids by inhibiting the expression of INDO. Our data showed that the expression levels of INDO and CTLA4 increased in the model group, and decreased after the treatment of THSW, indicating that THSW inhibited the expression of INDO and CTLA4, thus inhibiting the development of UL. This result is consistent with our findings. These results suggest that the therapeutic effect of THSW on UL may be related to the inhibition of JAK3 and STAT3 which can inhibit the expression of CTLA4 and INDO.

It is critical that the clinical treatment of uterine fibroids will change significantly in the future. The field needs to move towards the development of individualised treatment, as well as early intervention. In the recent decades, herbs and extractions from TCM, are gaining acceptance as promising complementary and alternative medicines for numerous diseases treatment [42-45]. The application of TCM can well achieve early intervention of symptoms and individualized treatment for different individuals. The application of TCM will have a positive impact on the intervention and treatment of uterine fibroids, which has also been confirmed in the experiment. In the next step, we should expand the clinical application of Taohong Siwu Decoction and conduct relevant cell experiments to obtain more accurate clinical guidance data.

\section{CONCLUSION}

The results showed that THSW could significantly improve the macroscopic pathological state of uterus in UL rats. The expression levels of JAK3 and STAT3, the major signal transduction molecules of cytokines and growth factors, were decreased in utero. It also reduced the expression of INDO and CTLA4. It was found that THSW could treat UL by inhibiting the activation of JAK3 and STAT3 and the expression of INDO/CTLA4. These data may provide a new way of thinking and treatment for laying a foundation for further study on the treatment of UL.
Abbreviations
TCM: Traditional Chinese medicine
THSW: Taohong Siwu decoction
$\boldsymbol{U} \boldsymbol{L}:$ uterine leiomyoma
JAK3 : Janus Kinase 3
STAT3: Signal transducer and activator of transcription 3 
SD: Sprague Dawley

CTLA4 : cytotoxic T lymphocyte associated antign 4

INDO : indoleamine 2, 3-dioxygenase 1

\section{Ethics Statement}

331 The animal study was reviewed and approved by the Animal Ethical Committee at the Chengdu University of Traditional Chinese Medicine.

\section{Consent for publication}

Not applicable.

\section{Data Availability Statement}

The datasets used and analysed during the current study are available from the corresponding author on reasonable request.

\section{Competing interests}

The authors declare that they have no competing interests.

\section{Funding}

This work was supported by the National Natural Science Foundation of China (Nos. 81001668 \&

\section{Author Contributions}

LL and SS conceived and designed the experiment. LL and SS carried out the experiment. LL retrieved the relevant literature and wrote the manuscript. LL and XX analyzed the experimental data and drew pictures. FP, LL, CY, LZ and TZ provided helpful comments and revised the manuscript. All authors contributed to the article and approved the submitted version.

\section{Acknowledgements}

358 I would like to extend my sincere thanks to all those who have helped me make this thesis possible and better. I also would like to extend my sincere thanks to all fundings.

\section{References}

362 [1] Holdsworth-Carson, S. J., Zaitseva, M., Vollenhoven, B. J. \& Rogers, P. A. W. Clonality of smooth muscle and fibroblast cell populations isolated from human and myometrial tissues. Mol Hum Reprod. (2014) 20:250-259.doi:10.1093/molehr/gat083

[2] Al-Hendy, A., Myers, E. R. \& Stewart, E. Uterine Fibroids: Burden and Unmet Medical Need. Semin Reprod Med. (2017) 35:473-480.doi:10.1055/s-0037-1607264

[3] Stewart, E. A., Laughlin-Tommaso, S. K., Catherino, W. H., Lalitkumar, S., Gupta, D. \& 

Vollenhoven, B. Uterine fibroids. Nat Rev Dis Primers. (2016) 2:16043.doi:10.1038/nrdp.2016.43

[4] Ma, L., Xie, S., Zhu, Y. \& Cao, L. Research progress of uterine fibroids. Reproduction and Contraception. (2007) 27:679-683+687

[5] Shen, T.-C., Yang, C.-Y., Huang, Y.-J., Lin, C.-L. \& Sung, F.-C. Risk of depression in patients with uterine leiomyoma: A nationwide population-based cohort study. Journal of Affective Disorders. (2017) 213:126-130.doi:10.1016/j.jad.2017.02.020

[6] Josephine, M., Michael, F., Patricia, T., James, S. \& Janeen, D. The economic impact of uterine fibroids in the United States:a summary of published estimates. Journal of Women's Health. (2005) 14:692-703.doi:10.1089/jwh.2005.14.692

[7] Borahay, M. A., Al-Hendy, A., Kilic, G. S. \& Boehning, D. Signaling pathways in leiomyoma: understanding pathobiology andimplications for therapy. Molecular Medicine. (2015) 21:242-256.doi:10.2119/molmed.2014.00053

[8] Shen, Y., Wu, Y., Lu, Q., Zhang, P. \& Ren, M. Transforming growthfactor-b signaling pathway cross-talking with ERa signaling pathway on regulating the growth of uterine leiomyomaactivated by phenolic environmental estrogens in vitro. Tumor Biology. (2016) 37:455-462.doi:10.1007/s13277-015-3813-4

[9] Bulun, S. E., Moravek, M. B., Yin, P., Ono, M., 5th, J. S. C., Dyson, M. T., et al. Uterine leiomyoma stem cells: linking progester-one to growth. Seminars in Reproductive Medicine (2015) 33:357-365.doi:10.1055/s-0035-1558451

[10] Borah, B. J., Laughlin-Tommaso, S. K., Myers, E. R., Yao, X. \& Stewart, E. A. Association between patient characteristics and treatment procedure among patients with uterine leiomyomas. Obstetrics \& Gynecology. (2016) 127:67-77.doi:10.1097/AOG.0000000000001160

[11] Gliklich, R. E., Leavy, M. B. \& Velentgas, P. Identification of future research needs in the comparative management of uter-ine fibroid disease: a report on the priori-ty-setting process, preliminary data anal-ysis, and research plan. Agency for Healthcare Research and Quality. 2011

[12] Hanafi, M. Predictors of leiomyoma recurrence after myomectomy. Obstetrics \& Gynecology. (2005) 105:877-881.doi:10.1097/01.AOG.0000156298.74317.62

[13] Merrill, R. M. Hysterectomy surveillance in the United States, 1997 through 2005. Medical Science Monitor. (2008) 14:CR24-31

[14] Zhou, J. \& Qu, F. Treating gynaecological disorders with traditional Chinese medicine: a review. African journal of traditional, complementary, and alternative medicines : AJTCAM. (2009) 6:494-517.doi:10.4314/ajtcam.v6i4.57181

[15] Zhao, H., Li, Y., Xu, Q., Peng, F., Zhao, J., Webb, R. C., et al. Establishment of a rat model for uterine leiomyomas based on Western and traditional Chinese medicine theories. Brazilian Journal of Medical and Biological Research. (2018) 51:e7627.doi:10.1590/1414-431X20187627

[16] Jiang, P., Wang, X., Dong, C., Bao, Y. \& Quan, J. Research on the correlation and progress of JAK3 in clinical application (review); proceedings of the Chinese Journal of Modern Medicine, F, 2020-09-17 [C].

[17] Raivola, J., Hammarén, H. M., Virtanen, A. T., Bulleeraz, V., Ward, A. C. \& Silvennoinen, O. Hyperactivation of Oncogenic JAK3 Mutants Depend on ATP Binding to the Pseudokinase Domain. Frontiers in Oncology. (2018) 8:560.doi:10.3389/fonc.2018.00560

[18] Leonard, W. J. \& O'Shea, J. J. Jaks and STATs: biological implications. Annual Review of 
Immunology. (1998) 16:293-322.doi:10.1146/annurev.immunol.16.1.293

[19] Ribas, A., Camacho, L. H., Lopez-Berestein, G., Pavlov, D., Bulanhagui, C. A., Millham, R., et al. Antitumor activity in melanoma and anti-self responses in a phase I trial with the anti-cytotoxic $\mathrm{T}$ lymphocyte-associated antigen 4 monoclonal antibody CP-675,206. Journal of Clinical Oncology. (2005) 23:8968-8977.doi:10.1200/JCO.2005.01.109

[20] Malemud, C. J. The role of the JAK/STAT signal pathway in rheumatoid arthritis. Therapeutic Advances in Musculoskeletal Disease. (2018) 10:117-127.doi:10.1177/1759720X18776224

[21] Kortylewski, M., Kujawski, M., Wang, T., Wei, S., Zhang, S., Pilon-Thomas, S., et al. Inhibiting Stat3 signaling in the hematopoietic system elicits multicomponent antitumor immu-nity. Nature Medicine. (2005) 11:1314-1321.doi:10.1038/nm1325

[22] Han, H., Li, D., Qian, R., Xu, X., Huang, Y., Ni, J., et al. To explore the mechanism of Lichong Decoction in inhibiting human uterine fibroid cells from the perspective of whole gene expression profile. Global Chinese Medicine. (2015) 8:290-295

[23] Xue, C., Xie, J., Zhao, D., Lin, S., Zhou, T., Shi, S., et al. The JAK/STAT3 signallingpathway regulated angiogenesis in an endothelial cell/adi-pose-derived stromal cell co-culture, 3D gel model. Cell Proliferation. (2017) 50:e12307.doi:10.1111/cpr.12307

[24] Shen, T.-C., Yang, C.-Y., Huang, Y.-J., Lin, C.-L. \& Sung, F.-C. Risk of depressionin patients with uterine leiomyoma: A nationwide population-based cohort study. Journal of Affective Disorders. (2017) 213:126-130.doi:10.1016/j.jad.2017.02.020

[25] Mauskopf, J., Flynn, M., Thieda, P., Spalding, J. \& Duchane, J. The economic impact of uterine fibroids in the United States:a summary of published estimates. Journal of Women's Health. (2005) 14:692-703.doi:10.1089/jwh.2005.14.692

[26] Cardozo, E. R., Clark, A. D., Banks, N. K., Henne, M. B., Stegmann, B. J. \& Segars, J. H. The estimated annual cost of uterine leiomyomata in the United States. American Journal of Obstetrics and Gynecology. (2012) 206:211.e211-211.e219.doi:10.1016/j.ajog.2011.12.002

[27] Meng, W. \& Zhao, W. Study on the effect of the method of replenishing qi and removing stasis on the expression of protein related to proliferation and apoptosis of cultured uterine fibroid cells in vitro. Chinese Journal of Traditional Chinese Medicine. (2008).doi:10.13193/j.archtcm.2008.02.15.mengw.038

[28] Yao, J. Acupuncture theory of promoting blood circulation and removing stasis and its clinical application. Zhongguo Zhen Jiu = Chinese acupuncture \& moxibustion. (2015) 35:389-392

[29] Wang, Z., Xiao, H., Li, X., Yao, X., Miao, J., Cui, J., et al. Research progress on pharmacological action of taohong siwu decoction. Modern traditional Chinese medicine. (2021) 41:22-28.doi:10.13424/j.cnki.mtcm.2021.02.005

[30] Li, S. Effect of taohong siwu decoction on uterine fibroids and its effect on serum sex hormone levels. Clinical medicine research and practice. 3:122-123.doi:10.19347/j.cnki.2096-1413.201812058

[31] Kortylewski, M., Kujawski, M., Wang, T., Wei, S., Zhang, S., Pilon-Thomas, S., et al. Inhibiting Stat3 signaling in the hematopoietic system elicits multicomponent antitumor immunity. Nature Medicine. (2005) 11:1314-1321.doi:10.1038/nm1325

[32] Liu, J.-B., Chen, D., Bao, T.-T., Fan, F.-T. \& Yu, C. The anticancer effects of atractylenolide III associate with the downregulation of Jak3/Stat3-dependent IDO expression. Frontiers in 
Pharmacology. (2020) 10:1505.doi:10.3389/fphar.2019.01505

[33] Wang, Y., Xu, M. \& Xiu, W. Experimental study of tanshinone combined with emodin inhibiting proliferation and migration of colorectal cancer cells through JAK/STAT3 signaling pathway. Progress of modern general surgery in China. (2020) 32:679-682+687

[34] Liu, M. Curcumin mediates JAK/STAT3 signaling pathway to regulate apoptotic migration and invasion of giant cell tumors of bone. Guangzhou. Southern Medical University (2019).

[35] Asher, S., Hannah, B.-B., Eyal, M., Neri, L., Y, K. B. \& Nathan, R. Inhibition of leiomyoma cell proliferation in vitro by genistein and the protein tyrosine kinase inhibitor TKS050. Fertility and Sterility. (2007) 87:127-135.doi:10.1016/j.fertnstert.2006.05.056

[36] Miguel, F. D., Lee, S. O., Onate, S. A. \& Gao, A. C. Stat3 enhances transactivation of steroid hormone receptors. Nuclear Receptor. (2003) 1:3.doi:10.1186/1478-1336-1-3

[37] Li, C. Expression of IL-6/STAT3/Cyclin D1 and VEGF signaling pathways in uterine fibroids. Hefei. Anhui Medical University, (2009).

[38] Dermani, F. K., Samadi, P., Rahmani, G., Kohlan, A. K. \& Najafi, R. PD-1/PD-L1 immune checkpoint: Potential target for cancer therapy. Journal of Cellular Physiology. (2019) 234:1313-1325.doi:10.1002/jcp.27172

[39] Fallarino, F. \& Grohmann, U. Using an Ancient Tool for Ig-niting and Propagating Immune Tolerance: IDO as an In-ducer and Amplifier of Regulatory T Cell Functions. Current Medicinal Chemistry. (2011) 18:2215-2221.doi:10.2174/092986711795656027

[40] Wing, K., Onishi, Y., Prieto-Martin, P., Yamaguchi, T., Miyara, M., Fehervari, Z., et al. CTLA-4 control over Foxp3+ regulatory $\mathrm{T}$ cell function. Science. (2008) 322:271-275.doi:10.1126/science. 1160062

[41] Sho, M., Salama, A. D., Yamada, A., Najafian, N. \& Sayegh, M. H. Physiologic regulation of alloimmune responses in vivo: the role of CTLA4 and TH1/TH2 cytokines. Transplantation Proceedings. (2001) 33:3826-3828.doi:10.1016/s0041-1345(01)02620-3

42 Peng, F., Xiong, L. \& Peng, C. (-)-Sativan Inhibits Tumor Development and Regulates miR-200c/PD-L1 in Triple Negative Breast Cancer Cells. Front. Pharmacol. 11, 12, doi:10.3389/fphar.2020.00251 (2020).

[43] Peng, F., Tang, H., Du, J., Chen, J. \& Peng, C. Isoliquiritigenin Suppresses EMT-Induced Metastasis in Triple-Negative Breast Cancer through miR-200c/C-JUN/ Formula: see text -Catenin. The American journal of Chinese medicine 49, 505-523, doi:10.1142/s0192415x21500233 (2021).

[44] Peng, F. et al. Isoliquiritigenin Derivative Regulates miR-374a/BAX Axis to Suppress Triple-Negative Breast Cancer Tumorigenesis and Development. Front. Pharmacol. 11, 11, doi:10.3389/fphar.2020.00378 (2020).

[45] Wang, L., Peng, F., Peng, C. \& Du, J.-R. Gut Microbiota in Tumor Microenvironment: A Critical Regulator in Cancer Initiation and Development as Potential Targets for Chinese Medicine. The American journal of Chinese medicine, 1-18, doi:10.1142/s0192415x21500270 (2021).

\section{Figure legends}

Figure 1. Schematic design of the experiment. 
Figure 2. Changes in the size of the uterus. Data are reported as mean $\pm \mathrm{SD} .{ }^{*} p<0.05, * * p<0.01$, $* * * p<0.001$ compared with control group; ${ }^{*} p<0.05,{ }^{\# \#} p<0.01,{ }^{\# \#} p<0.001$ compared with model group_(one-way ANOVA and LSD test).

Figure 3. weight changes of rat.Data are reported as mean \pm SD. ${ }^{*} p<0.05$, ${ }^{*} p<<0.01$, $* * * p<$ 0.001 compared with control group; ${ }^{\#} p<0.05,{ }^{\# \#} p<0.01$, ${ }^{\# \#} p<0.001$ compared with model group (one-way ANOVA and LSD test).

Figure 4. Effects of THSW on the Appearance of the Uterus. (A) control group; (B) model group; (C) positive group; (D) THSW (18g/ml); (E) THSW (9g/ml); (F) THSW (4.5g/ml).

Figure 5. Histopathologic examinations of uteruses. (A) control group; (B) model group; (C) positive group; (D) THSW (18g/ml); (E) THSW (9g/ml); (F) THSW (4.5g/ml).

Figure 6. Effect of THSW on activation of JAK3 in uterus. Data are reported as mean \pm SD. ${ }^{*} p<0.05$, ${ }^{* *} p<0.01,{ }^{* * *} p<0.001$ compared with control group; ${ }^{\#} p<0.05,{ }^{\# \#} p<0.01,{ }^{\# \#} p<0.001$ compared with model group_(one-way ANOVA and LSD test).

Figure 7. Effect of THSW on activation of STAT3 in uterus. Data are reported as mean \pm SD. $*^{*}<0.05$, ${ }^{* *} p<0.01, * * * p<0.001$ compared with control group; ${ }^{\#} p<0.05,{ }^{\# \#} p<0.01,{ }^{\# \#} p<0.001$ compared with model group (one-way ANOVA and LSD test).

Figure 8. Effects of THSW on the expression of INDO and CTLA4 in uterus. (A-C) Representative western blotting band of INDO, CTLA4 Data are reported as mean \pm SD. ${ }^{*} p<0.05, * * p<0.01,{ }^{* * *} p$ $<0.001$ compared with control group; ${ }^{\#} p<0.05,{ }^{\#} p<0.01,{ }^{\# \#} p<0.001$ compared with model group (one-way ANOVA and LSD test). 


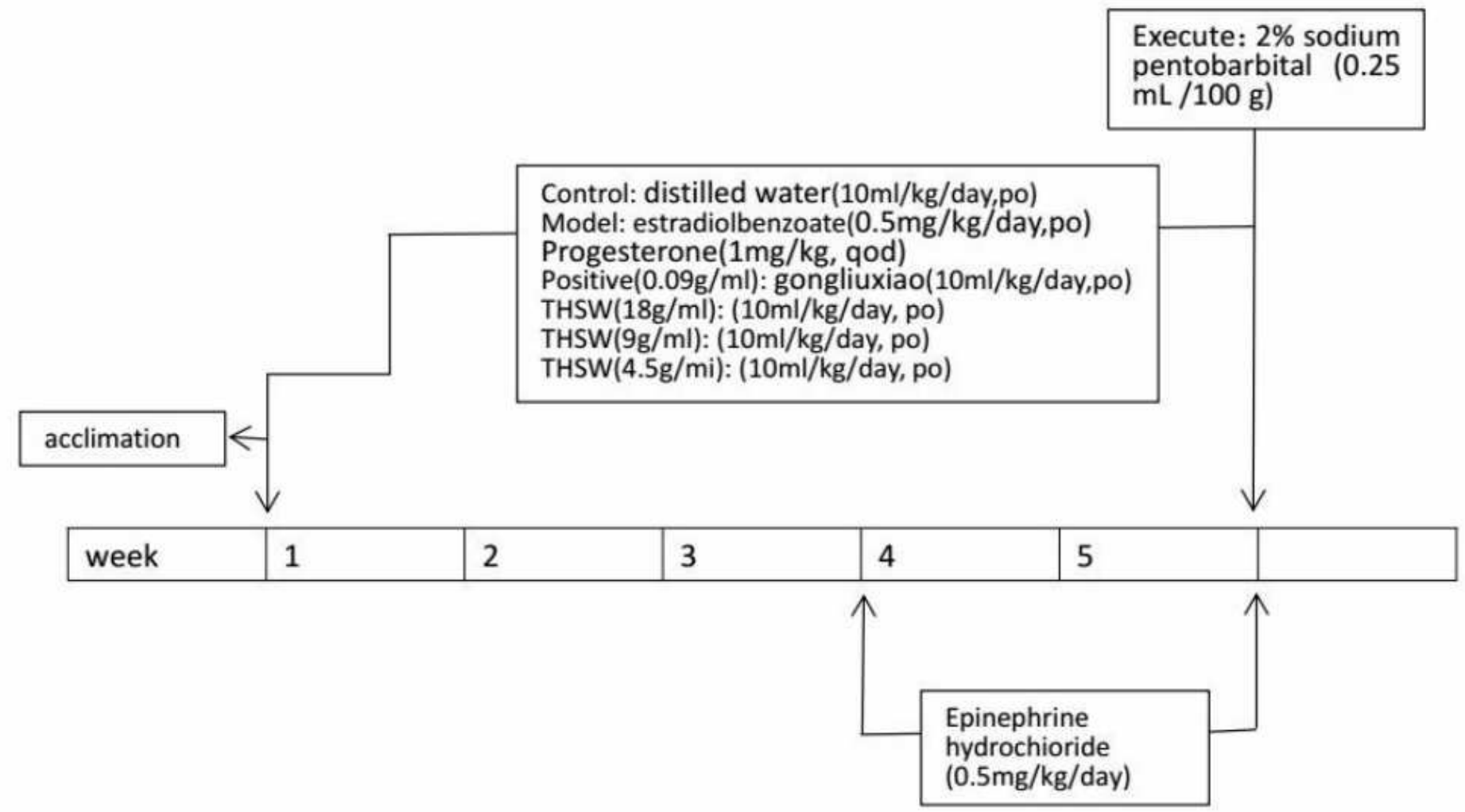

Figure 1

Schematic design of the experiment

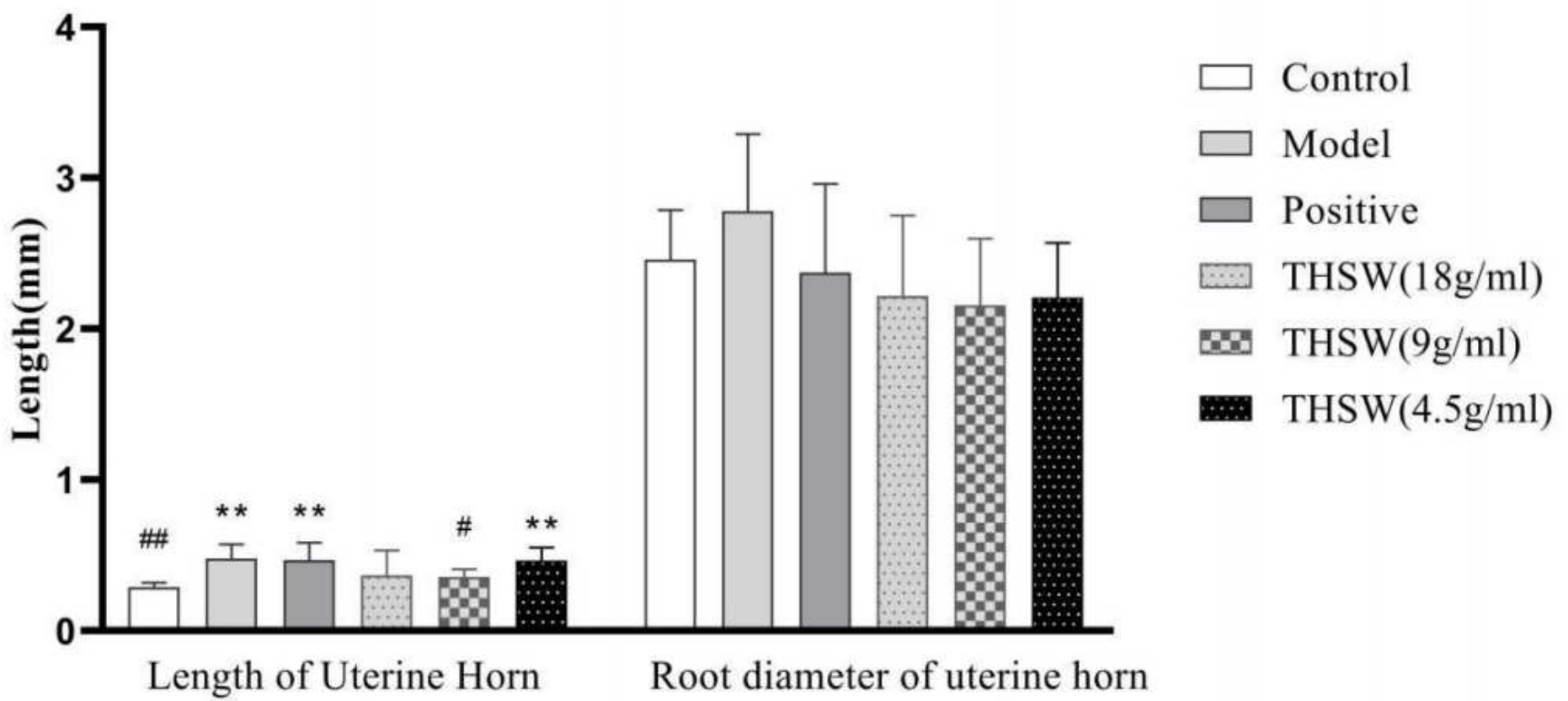


Figure 2

Changes in the size of the uterus. Data are reported as mean $\pm \mathrm{SD}$. ${ }^{\star} \mathrm{P}<0.05,{ }^{\star *} \mathrm{P}<0.01,{ }^{\star \star *} \mathrm{P}<0.001$ compared with control group; \#P $<0.05$, \#\#P $<0.01$, \#\# $\#$ P 0.001 compared with model group (one-way ANOVA and LSD test).

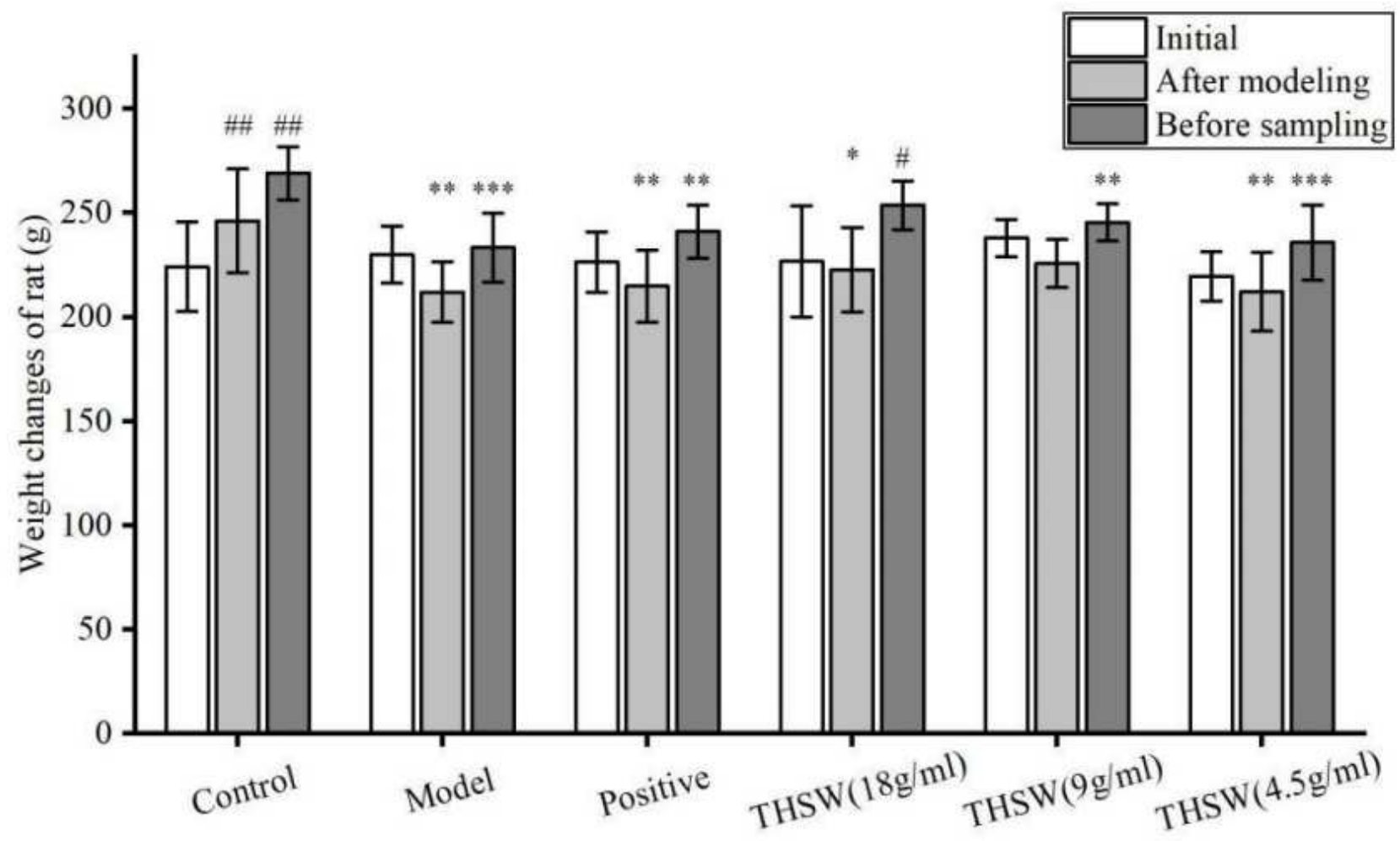

Figure 3

Weight changes of rats. Data are reported as mean $\pm S D$. ${ }^{*} P<0.05$, ${ }^{\star *} P<0.01,{ }^{*} * \mathrm{P}<0.001$ compared with control group; $\# \mathrm{P}<0.05, \# \# \mathrm{P}<0.01$, \#\#\#P<0.001 compared with model group (one-way ANOVA and LSD test) 

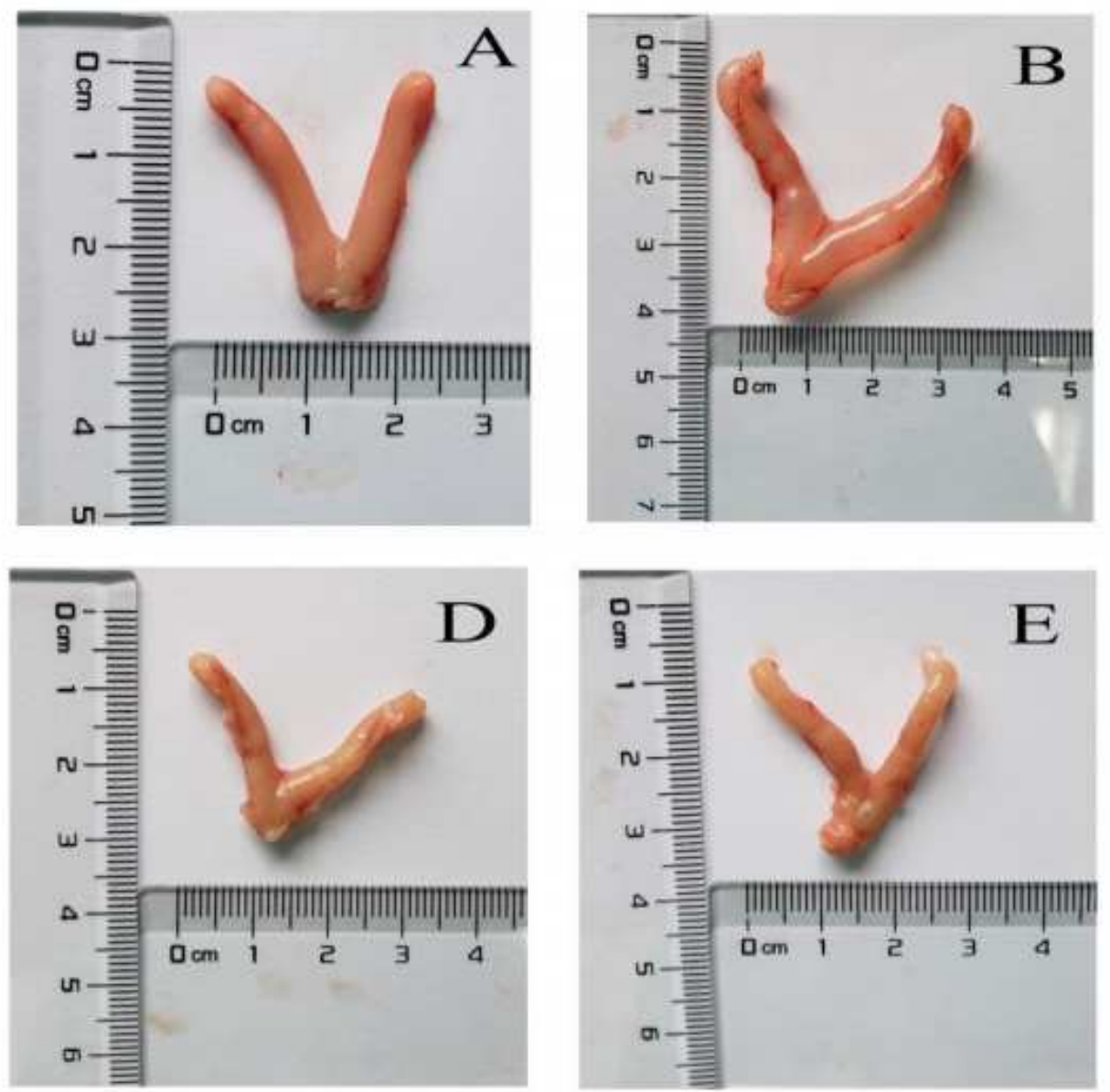
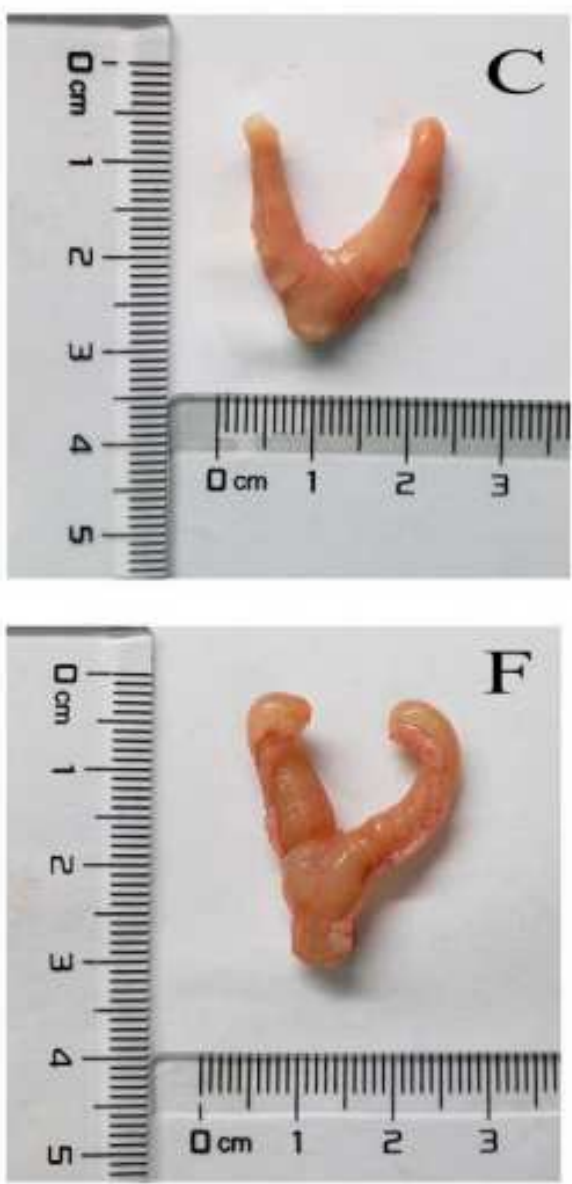

\section{Figure 4}

Effects of THSW on the Appearance of the Uterus. (A) control group; (B) model group; (C) positive group; (D) THSW (18g/ml) group; (E) THSW ( $9 \mathrm{~g} / \mathrm{ml})$ group; (F) THSW $(4.5 \mathrm{~g} / \mathrm{ml})$
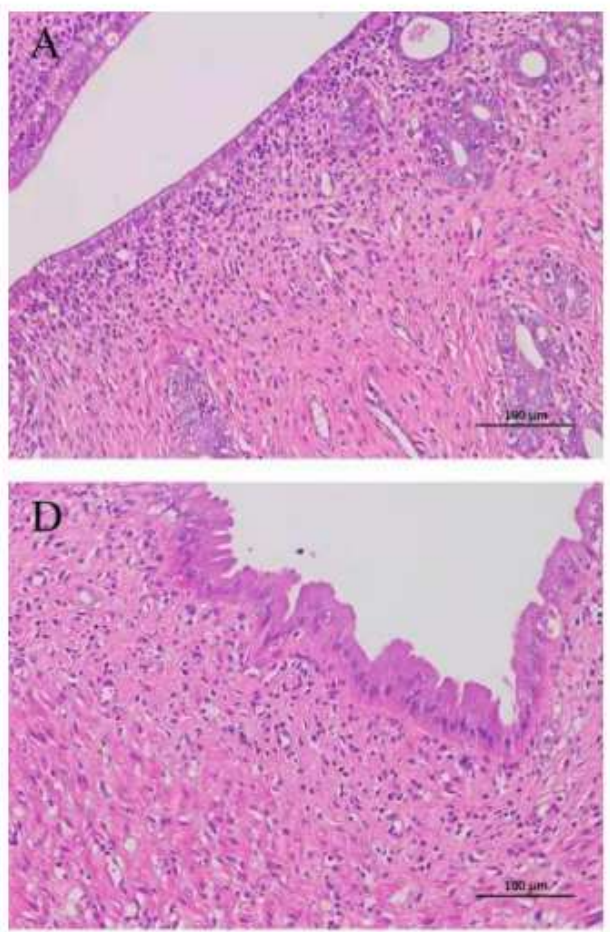
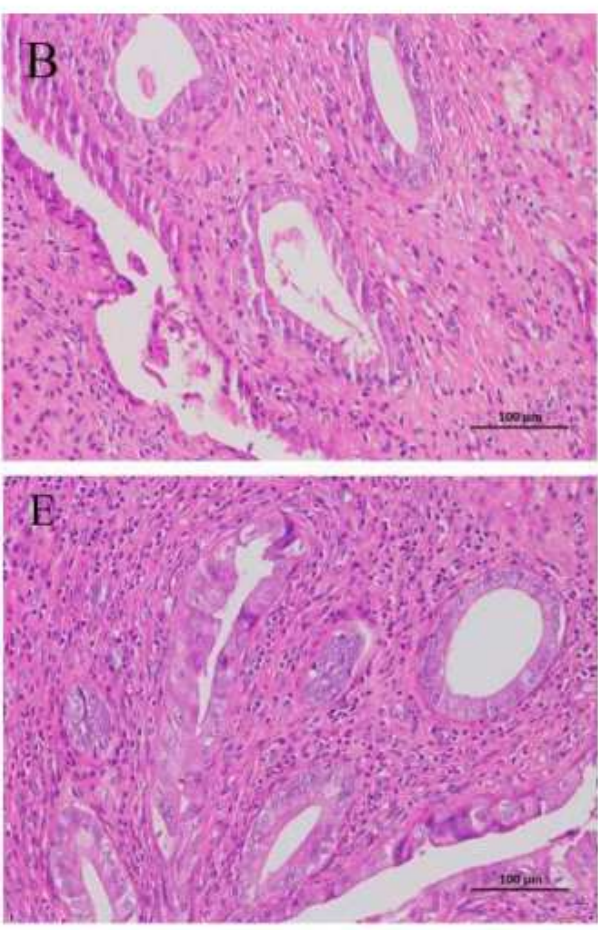
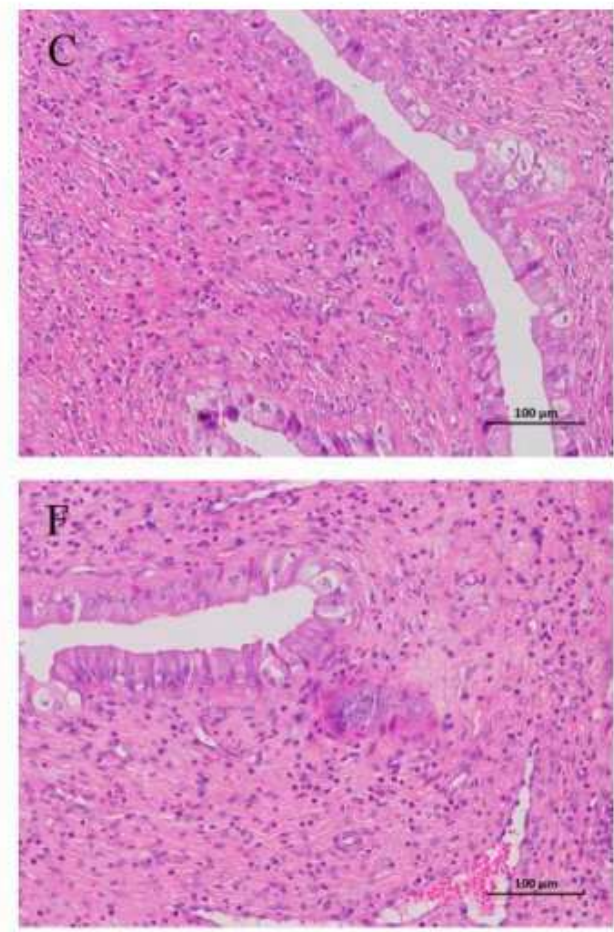
Figure 5

Histopathologic examinations of uteruses. (A) control group; (B) model group; (C) positive group; (D) THSW (18g/ml) group; (E) THSW (9g/ml) group; (F) THSW $(4.5 \mathrm{~g} / \mathrm{ml})$ gro

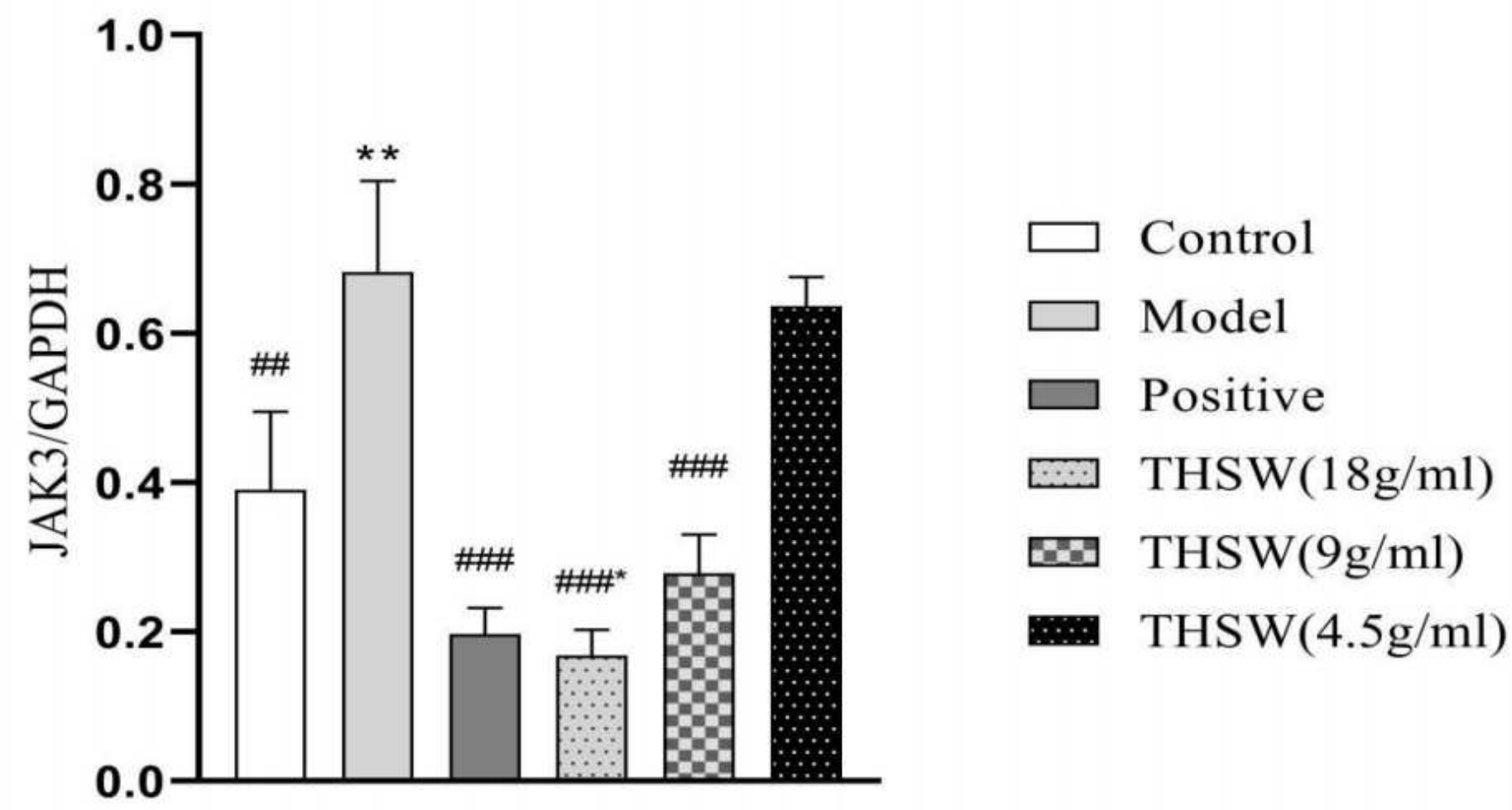

Figure 6

Effect of THSW on activation of JAK3 in uterus. Data are reported as mean $\pm S D$. ${ }^{*} P<0.05$, $* * P<0.01$, $\star \star \star \mathrm{P}<0.001$ compared with control group; \#P $<0.05$, \#\#P $<0.01$, \#\#\# $<0.001$ compared with model group (one-way ANOVA and LSD test). 


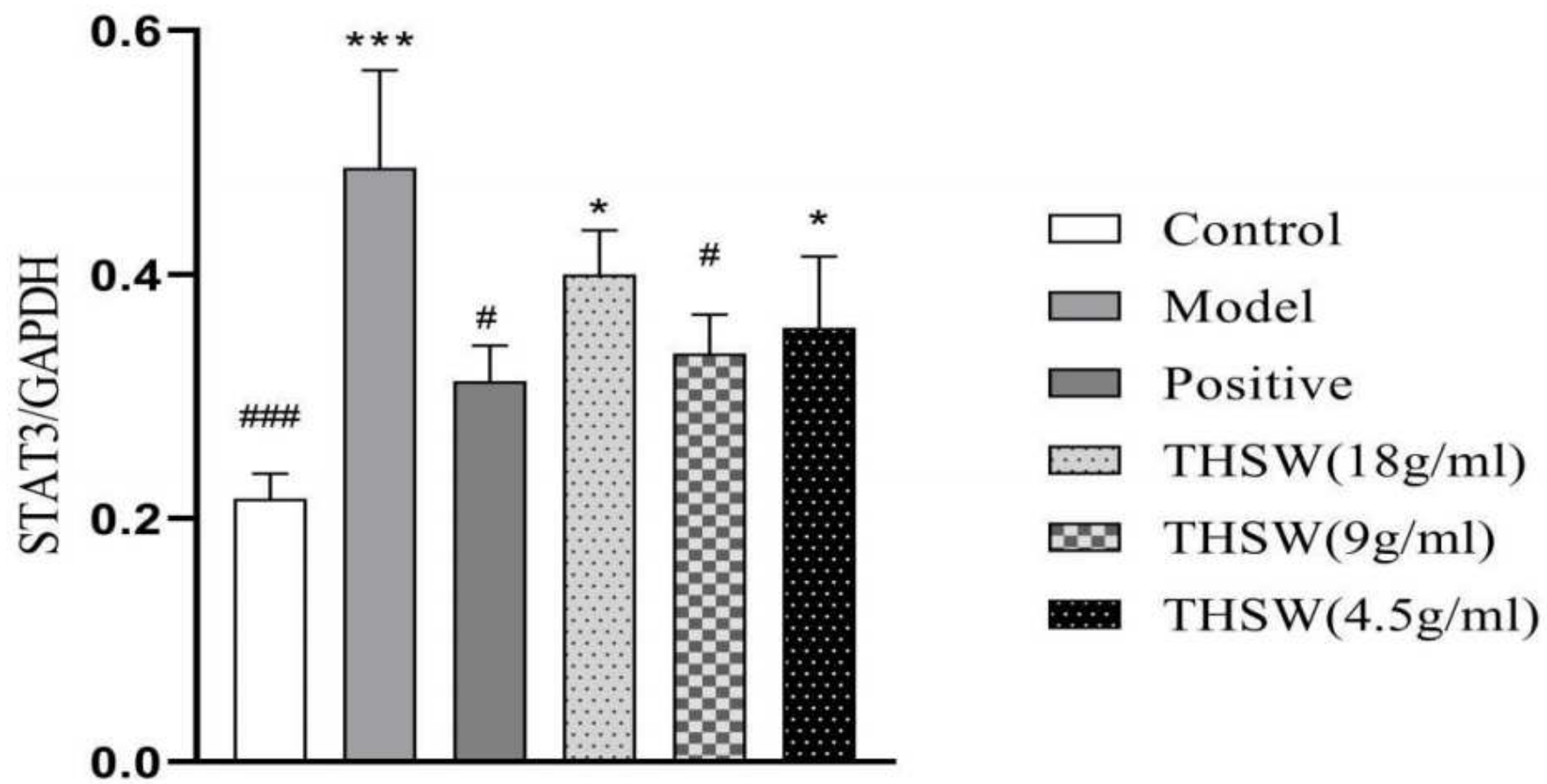

Figure 7

Effect of THSW on activation of STAT3 in uterus. Data are reported as mean \pm SD. ${ }^{*} P<0.05, * * P<0.01$, $\star \star \star P<0.001$ compared with control group; \#P $<0.05$, \#\#P $<0.01$, \#\#\#P $<0.001$ compared with model group (one-way ANOVA and LSD test). 


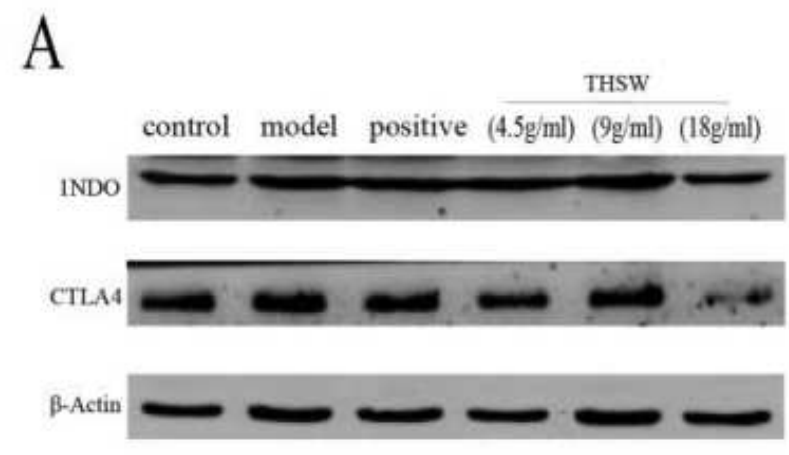

B

C
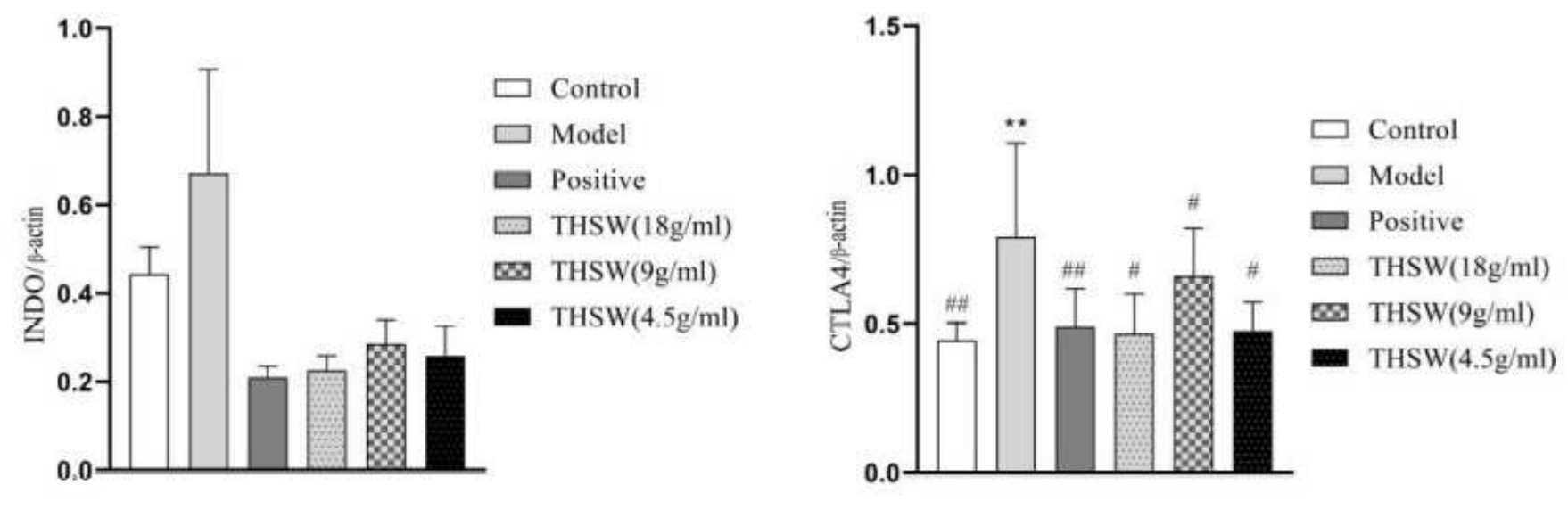

Figure 8

Effects of THSW on the expression of INDO and CTLA4 in uterus. (A-C) Representative western blotting band of INDO, CTLA4 Data are reported as mean $\pm S D$. ${ }^{*} P<0.05$, $* * P<0.01, * \star * P<0.001$ compared with control group; $\# \mathrm{P}<0.05, \# \# \mathrm{P}<0.01$, \#\#\#P $<0.001$ compared with model group (one-way ANOVA and LSD test).

\section{Supplementary Files}

This is a list of supplementary files associated with this preprint. Click to download.

- SupplenmentaryTable11.docx 